\title{
MONSTARS ON GLACIERS
}

\author{
By J. F. NYE \\ (H. H. Wills Physics Laboratory, University of Bristol, Bristol BS8 1TL, England)
}

\begin{abstract}
Isotropic points are structurally stable features of any complicated field of stress or strain-rate, and therefore will almost always be present on the surface of a glacier. A given isotropic point for strain-rate will belong to one of six different classes, depending on the pattern (lemon, star, or monstar) of principal directions and the contours (ellipses or hyperbolas) of constant principal strain-rate values in its neighbourhood. The central isotropic point on a glacier should theoretically have a monstar pattern, but the contours around it may sometimes be elliptic and sometimes hyperbolic. Nearby, but not coincident with it, there will be an isotropic point for stress. This will also have a monstar pattern but, in contrast to the strain-rate point, the contours around it must be hyperbolic. Published examples are consistent with these conclusions. In addition to isotropic points for strainrate a glacier surface will contain isolated points of pure shear; these also can be classified into six different types. Stable features of this kind give information about the essential structure of a tensor field and form useful points of comparison between observation and numerical simulation.
\end{abstract}

RÉSumÉ. "Monstars» sur les glaciers. Des points isotropiques sont des caractères structurellement stables de tout champ compliqué de contraintes ou de déformation, et seront donc presque toujours présents à la surface d'un glacier. Un point isotropique donné pour les vitesses de déformation sera de l'une des six classes différentes, selon la forme (en lemon= «citron», en star= «étoile» ou monstar= «citronétoile») des directions principales et des iso-lignes (ellipses ou hyperbolas) de valeur constante des vitesses principales de déformation dans leur voisinage. Le point isotropique central sur un glacier devrait théoriquement avoir un comportement monstar, mais les iso-lignes autour de lui peuvent être soit elliptiques soit hyperboliques. Proche, mais non en coïncidence avec lui, sera le point isotropique pour les contraintes. Il aura aussi un comportement monstar mais contrairement au point isotropique pour les vitesses de déformation les iso-lignes autour de lui doivent être hyperboliques. Les exemples publiès sont en bon accord avec ces conclusions. En plus des points isotropiques pour les vitesses de déformation une surface de glacier contiendra des points isolés de pur cisaillement, ceux-ci peuvent également être classés en six types différents. Des caractéristiques stables de cette sorte donnent des informations sur la structure essentielle du champ d'un tenseur et forment d'utiles points de comparaison entre les observations et la simulation numérique.

ZuSAmmenfassung. „Monstars” auf Gletschern. Isotropische Punkte sind strukturell stabil Erscheinungen eines jeden komplizierten Spannungs- und Deformationsfeldes; es wird sie deshalb fast immer auf der Oberfläche eines Gletschers geben. Ein bestimmter isotropischer Punkt für die Deformationsrate wird zu einer von sechs verschiedenen Klassen gehören, je nach dem Muster (lemon=,Zitrone", star =,,Stern" oder monstar = .Zitronenstern”) der Hauptrichtungen und den Isolinien (Ellipsen oder Hyperbeln) der konstanten Hauptdeformationsraten in seiner Nachbarschaft. Der zentrale isotropische Punkt auf einem Gletscher sollte theoretisch ein Monstar-Muster haben, aber die Isolinien um ihn herum können manchmal elliptisch und manchmal hyperbolisch sein. In seiner Nähe, aber nicht mit ihm zusammenfallend, wird ein isotropischer Punkt für die Spannung liegen. Dieser wird ebenfalls ein Monstar-Muster besitzen, aber seine Isolinien müssen im Gegensatz zum ersten hyperbolisch verlaufen. Bekannt gewordene Beispiele stimmen mit diesen Schlüssen überein. Zusätzlich zu isotropischen Punkten der Deformationsrate wird eine Gletscheroberfläche isolierte Punkte mit reiner Scherung enthalten; auch diese können mit sechs verschiedenen Typen klassifiziert werden. Stabile Erscheinungen dieser Art geben Auskunft über die wesentliche Struktur eines Tensorfeldes und bilden nützliche Vergleichspunkte zwischen der Beobachtung und der numerischen Simulation.

\section{Genericity and Structural stability}

This paper is concerned with the field of stress and strain-rate on the surface of a glacier. Analytical treatments have to idealize the shape of the naturally irregular glacier boundaries to make them geometrically simple, and to simplify the real and complicated constitutive law for ice 
creep; in these respects the glacier problem is no different from any other in geophysics. Nonetheless it is possible to make certain precise statements about the stress and strain-rate fields, and other features of glaciers, which are independent of idealizations of this kind and rely on little more than continuity. Such statements apply to structural features that are generic, in the sense that they occur naturally without any special conditions being imposed.

An almost trivial example is the equilibrium line. If there is accumulation in one part of a glacier and ablation in another, there must be, somewhere, by continuity, an equilibrium line where the accumulation is zero. It has the important property that a small change in conditions cannot annihilate it; it will only be moved slightly. A feature with this property is said to be structurally stable.

Less trivial examples, not so widely recognized, are the isotropic points for stress and strainrate, described in outline in Nye (1981, Appendix B, p. 564-68), which we classify and discuss in this paper. Their structural stability means that they act as more or less permanent markers in a complicated (and changing) field. It is important to identify them, because their nature and positions represent information about the essential structure of the field, rather than inessential numerical detail. Now that it is becoming possible to model the flow of an irregularly shaped glacier numerically we need structural information of this kind. For example, we can ask how well the isotropic points observed in a real glacier agree in position with those in the numerical simulation, and whether they are of the predicted kind.

This paper pays special attention to a particular isotropic point that is expected to occur in virtually all glaciers, however complicated, and it suggests that it will be of the kind called monstar. If the point is not a monstar in a selected glacier one would look for a special reason. The analysis of isotropic points also has relevance to patterns of crevasses, in so far as these reflect the pattern of principal stress directions. Finally it should be said that, although the paper is focused on valley glaciers, the general ideas it expresses about genericity and structural stability (terms borrowed from mathematical singularity theory) and about isotropic points are equally applicable to ice sheets and ice shelves, to sea-ice regarded as a continuum, to moving snow cover, and indeed to any continuous two-dimensional distribution of stress or strain or strain-rate.

\section{ISOTROPIC POINTS ON GLACIERS}

At an isotropic point for strain-rate in the glacier surface, two conditions on the tensor components must be satisfied: $\dot{\varepsilon}_{x x}=\dot{\varepsilon}_{y y}$ and $\dot{\varepsilon}_{x y}=0$, where $\mathrm{O} x, \mathrm{O} y$ are axes in the surface. Each condition defines a line, or set of lines, in the surface, and therefore the two together define a point or set of points. If $\mathrm{O} x$ is taken as longitudinal down-glacier, $\dot{\varepsilon}_{x y}=0$ is satisfied on. a longitudinal line near the centre of the glacier; on the other hand $\dot{\varepsilon}_{x x}=\dot{\varepsilon}_{y y}$ will usually be satisfied on one or more roughly transverse lines; isotropic points will occur at intersections. For example, if the valley walls are roughly parallel, one of the transverse lines will probably be near the equilibrium line, where $\dot{\varepsilon}_{x x}$ is changing sign and $\dot{\varepsilon}_{y y}$ is small, and in this case an isotropic point will exist near the centre of the equilibrium line.

Mathematically, the isotropic conditions define points which must be expected to occur even in the most complicated glacier. In fact, the more complicated it is the more such points there will be.

The isotropic points of a general two-dimensional field of a symmetric second-rank tensor (such as strain-rate or stress) have recently been classified (Thorndike and others, 1978) and 
found to fall into six different categories. The classification depends on three properties possessed by a given isotropic point, namely, index, line, and contour, each property having one or other of two possible values. In principle this would give $2^{3}$ categories, but two of them demand incompatible conditions so there are in fact six possibilities. Taking strain-rate as an example, the index property of a given isotropic point is defined by imagining a small circuit around the point and noting how the cross of principal strain-rate directions rotates as one traverses the circuit once. It necessarily rotates by an integral multiple of $\pi$. If it rotates by $+\pi$ the index is defined to be $+\frac{1}{2}$, and if $-\pi$ then $-\frac{1}{2}$. Figure 1 shows three examples of patterns of trajectories of principal strain-rate. Figure $1 \mathrm{~b}$ has index $-\frac{1}{2}$, while Figure $1 \mathrm{a}$ and $\mathrm{c}$ have index $+\frac{1}{2}$. Other integral multiples of $\pi$ will not occur in natural glaciers at an arbitrary instant of time, but only in specially contrived theoretical models; they are structurally unstable; if they are observed it indicates an unresolved cluster of points with indices $\pm \frac{1}{2}$.

The line property is defined as 1 or 3 according to whether there are 1 or 3 straight strainrate trajectories emerging from the point (straight in the lowest order approximation). Thus the line property of Figure 1a is 1 while that of Figure $1 \mathrm{~b}$ and $\mathrm{c}$ is 3 . Since it is not possible to have simultaneously negative index and 1 line, the index and line properties together define three possible patterns, of which those in Figure 1 are symmetrical examples; they are called repectively lemon, star, and (because intermediate) monstar, the nomenclature being due to Berry and Hannay (1977), who describe and analyse the patterns in relation to the umbilic points of a general curved surface.

The contour property is not related to these patterns of trajectories but to the contours of equal magnitude of the two principal strain-rates near the isotropic point. These form two sets which are either both ellipses or both hyperbolas. In the first case the isotropic point for strainrate is said to be elliptic and in the second case hyperbolic. Each possible pattern, lemon, star, or monstar, can be either elliptic or hyperbolic; therefore there are, in all, six categories of isotropic point for strain-rate. Likewise, by substituting the word stress for strain-rate in the definitions, there are six different categories of isotropic point for stress.

In an anisotropic medium like a glacier an isotropic point for strain-rate will not be an isotropic point for stress, and vice versa. But in the approximation in which the flow law is isotropic the points will coincide. Moreover, in that case, since the principal axes for strain-rate and stress will be the same at any given point, the pattern of trajectories will be identical for

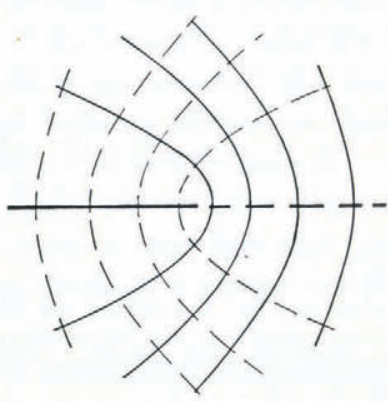

(a)

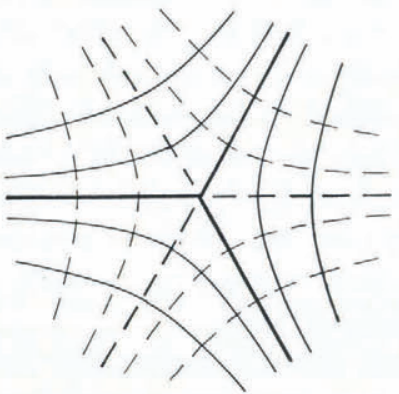

(b)

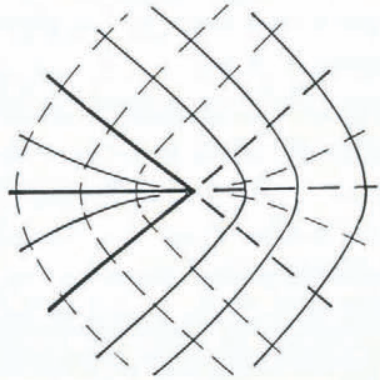

(c)

Fig. 1. Patterns of trajectories of principal strain-rate or stress directions around an isotropic point: (a) lemon, (b) star, (c) monstar. 
strain-rate and stress. Hence the pattern classification of a given isotropic point will be the same for both tensors. However, this is not true of the contour classification; a given isotropic point could be, for example, elliptic for strain-rate but hyperbolic for stress.

\section{Classification of the CENTRAL isotropic Point on a GlaCiER}

What kind of isotropic points should we expect on a glacier? The notion of structural stability referred to in $\S 1$ is important here. An isotropic point is structurally stable in the sense that a small perturbation in the field (caused by a change in the boundary conditions or in the constitutive law or simply by the passage of time) will not destroy it, but will merely move it and slightly distort its associated pattern. But the notion goes deeper than this, for the classification of a given isotropic point is also stable against small perturbation. This means that we can work with the simplest mathematical model without necessarily doing any violence at all to the real phenomenon.

If a glacier were straight, with no lateral variations $\left(\dot{\varepsilon}_{y y} \equiv 0\right)$ an isotropic point would be found on the centre-line $\left(\dot{\varepsilon}_{x y}=0\right)$ at the point of maximum velocity $\left(\dot{\varepsilon}_{x x}=0\right)$. In the real case, however, where $\dot{\varepsilon}_{y y} \not \equiv 0$, this central isotropic point will be displaced up or down the glacier, but typically it will always exist (unless it were displaced over the glacier boundary, which must be a very rare occurrence indeed). The classification of the isotropic point depends on the coefficients in the linear approximation to the strain-rate components about the point. With origin $\mathrm{O}$ at the point let us start by considering the approximation

$$
\dot{\varepsilon}=\left(\begin{array}{cc}
E-a x & -b y \\
-b y & E
\end{array}\right) \quad(0<a<b),
$$

where $E$ is the common value of $\dot{\varepsilon}_{x x}$ and $\dot{\varepsilon}_{y y}$ at O. In general, all three independent components will vary with both $x$ and $y$, but, out of six constants thus implied, we have selected $a$ and $b$ as representing the most important variations, their indicated signs and relative magnitudes being representative of most glaciers. Thorndike and others (1978, appendix 3) give three discriminants, involving the linear coefficients, whose signs decide the three properties involved in the classification. In this case they show that the point has index $+\frac{1}{2}$, and the line property is 3. The trajectories of principal strain-rate therefore form a monstar pattern (Figure 2b). However, the discriminant whose sign decides the contour classification is zero, signifying a degenerate case (parabolic). This raises an important point of principle which we have touched on already. We are concerned with what occurs typically on glaciers, and degenerate isotropic points do not occur typically in distributions of strain-rate unless there is some special constraint such as symmetry. They are structurally unstable. Our result implies that a small perturbation will make the point either elliptic or hyperbolic according to its sign. One possible perturbation is to put $\dot{\varepsilon}_{y y}=E+c x$ ( $c$ small); evaluation of the contour discriminant then shows that if $c<0$ the point is hyperbolic and if $\mathrm{c}>0$ elliptic. Physically, $c<0$ and $c>0$ correspond to flow-lines that are respectively concave and convex towards $\mathrm{O}$ (simple convergence or divergence merely affects the sign of $E$ ). This is just one of several types of perturbation that would break the degeneracy. The monstar pattern is typical and structurally stable in that it is unchanged by allowing small extra linear variations (for example, $\dot{\varepsilon}_{y y}=E+c x$, or $\dot{\varepsilon}_{x x}=E-a x+a^{\prime} y$ ); to avoid it would require either large extra linear variations or a violation of the condition $0<a<b$. Thus it is not absolutely impossible to have a different pattern, but if a different one were observed one 

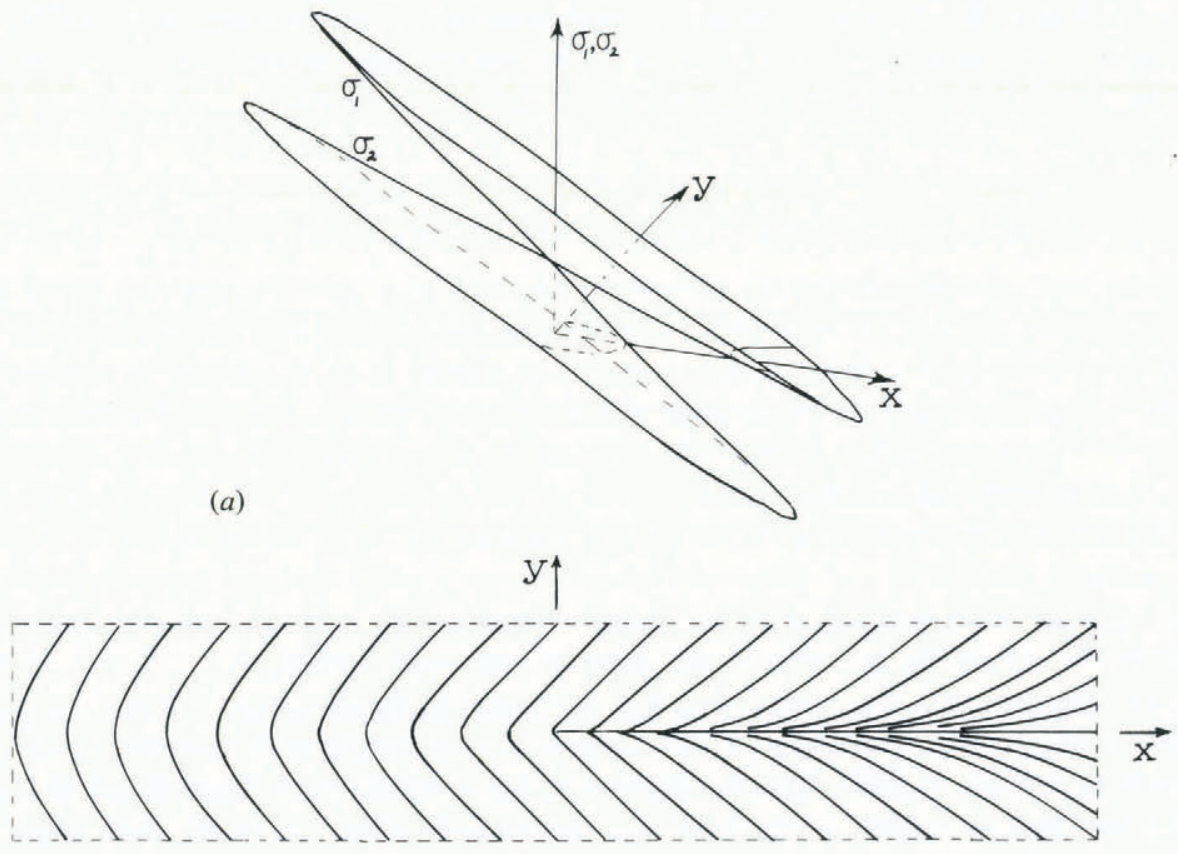

(b)

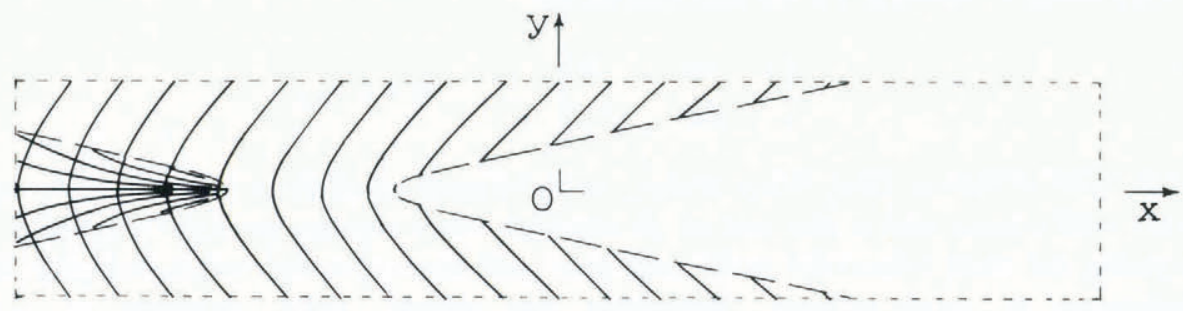

(c)

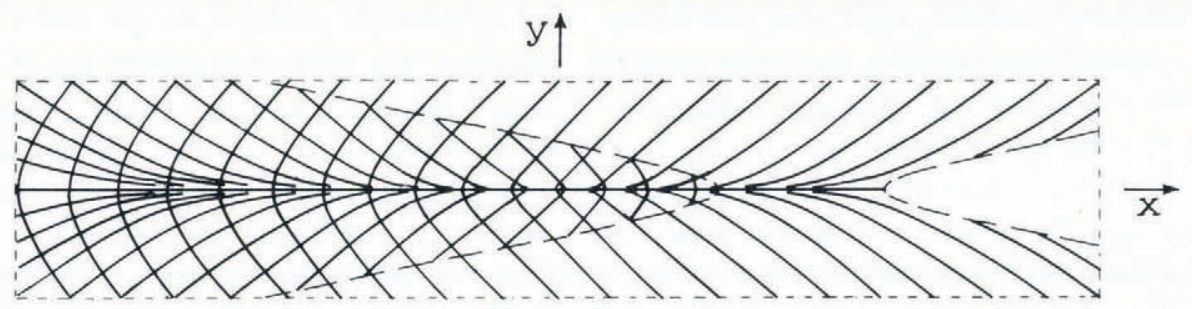

Fig. 2. (a) Magnitudes of the two principal stresses $\sigma_{1}, \sigma_{2}\left(\sigma_{1} \geqslant \sigma_{2}\right)$ plotted as functions of $x, y$; the apex of the cone is at $\sigma_{1}=\sigma_{2}=6 \mu E$. (b) Pattern of trajectories of the algebraically lesser principal strain-rate and stress corresponding to equation (1), with $b / a=7$; the straight-line trajectories through $O$ makes angles with $O x$ of $O$ and $\pm \tan ^{-1} \sqrt{ }\{1-(a / b)\}$, which in this case are 0 and $\pm 42.3^{\circ}$. (c) Crevasse pattern for a compressive isotropic point $(E<0)$ as might be formed by a narrowing glacier channel; only the immediate neighbourhood of the isotropic point, where the linear approximation holds, is shown. (d) The same for a tensile isotropic point $(E>0)$ associated with diverging flow; the hyperbola is the zero-level section in (a).

would wish to ask why. On the other hand, because the contour classification is borderline we must expect both hyperbolic and elliptic monstars in Nature-neither should surprise us. 
If the ice is assumed isotropic in its flow properties, $\mathrm{O}$ will be an isotropic point for stress as well as for strain-rate. Further, since the principal directions will now be the same for both tensors, their trajectory patterns will also be the same. Therefore $\mathrm{O}$ is a monstar for stress. However, its contour classification is different. To show this we start by using a linear-viscous flow law. Then the stress tensor $\sigma$ on $z=0$ is

$$
\sigma=2 \mu\left(\begin{array}{cc}
3 E-2 a x & -b y \\
-b y & 3 E-a x
\end{array}\right),
$$

where $\mu$ is the viscosity. This is obtained from Equation (1) by assuming incompressibility, so that $\dot{\varepsilon}_{z z}=-2 E+a x$, setting the stress deviator proportional to $\dot{\varepsilon}$, and adding a hydrostatic part to make the normal traction $\sigma_{z z}$ zero. Equilibrium with the body forces is secured by $z$ variations, which do not concern us. Evaluation of the appropriate discriminant shows that the contour classification is now hyperbolic, and since we are dealing with a stable property this conclusion will be unchanged if we make small perturbations either of the other possible linear variations or even of the linear-viscous flow law (for example, by adding a non-linear part).

More interestingly, if we allow the ice to be not too severely anisotropic, the isotropic point for stress will separate from that for strain-rate, but will remain hyperbolic monstar. Thus, as a general rule, whereas the isotropic point for strain-rate can be either elliptic or hyperbolic monstar, the nearby isotropic point for stress will be hyperbolic monstar.

Figure $2 \mathrm{~b}$ shows the monstar pattern made by the trajectories corresponding to the more compressive of the two principal stresses. When the values of the two principal stresses are plotted as functions of $x, y$ the resulting surface is a double cone (Figure 2a). If we assume that crevasses form perpendicular to the principal stress directions wherever the stress is tensile, the local pattern around $\mathrm{O}$ will depend on the sign of $E$. Figures $2 \mathrm{c}$ and $\mathrm{d}$ each show three regions, bounded by the two branches of a hyperbola, corresponding to zero, one, or two principal stresses tensile. The boundary is a hyperbola because, from the contour classification, the locus of a constant (here zero) principal stress value is a hyperbola, and it corresponds to a section of the cone in Figure 2a either above $(E<0)$ or below $(E>0)$ the apex. (It must be remembered, of course, that the crevasses one observes on a glacier have been carried away from the places where they were originally formed and have been rotated by the flow.)

The measured pattern of strain-rate directions in Figure 3 is an excellent illustration of these principles. The pattern is clearly monstar (compare Figure $2 b$ ), as expected; not, of course, a precisely symmetrical monstar but a monstar nonetheless, because such a pattern, with its three straight lines and positive index is structurally stable and evidently able to survive the perturbation from the ideal symmetrical example to the irregularites of Nature. The fact that the centre of the pattern is missing is just what one would expect, because there will necessarily be a region close enough to $\mathrm{O}$ where the principal directions are indistinguishable in the noise. Another example appears in Figure 3b of Hambrey and others (1980).

\section{RELATION TO GENERALIZED UMBILIC POINTS DEFINED By WhitNEY'S a DIRECTION}

Thorndike and others (1978) consider a measured instantaneous two-dimensional velocity field of sea ice, they recognize what they define as generalized umbilic points and then proceed to classify them. The classification is very close to what we have described for strain-rate but involves a subtle distinction which we now discuss. 


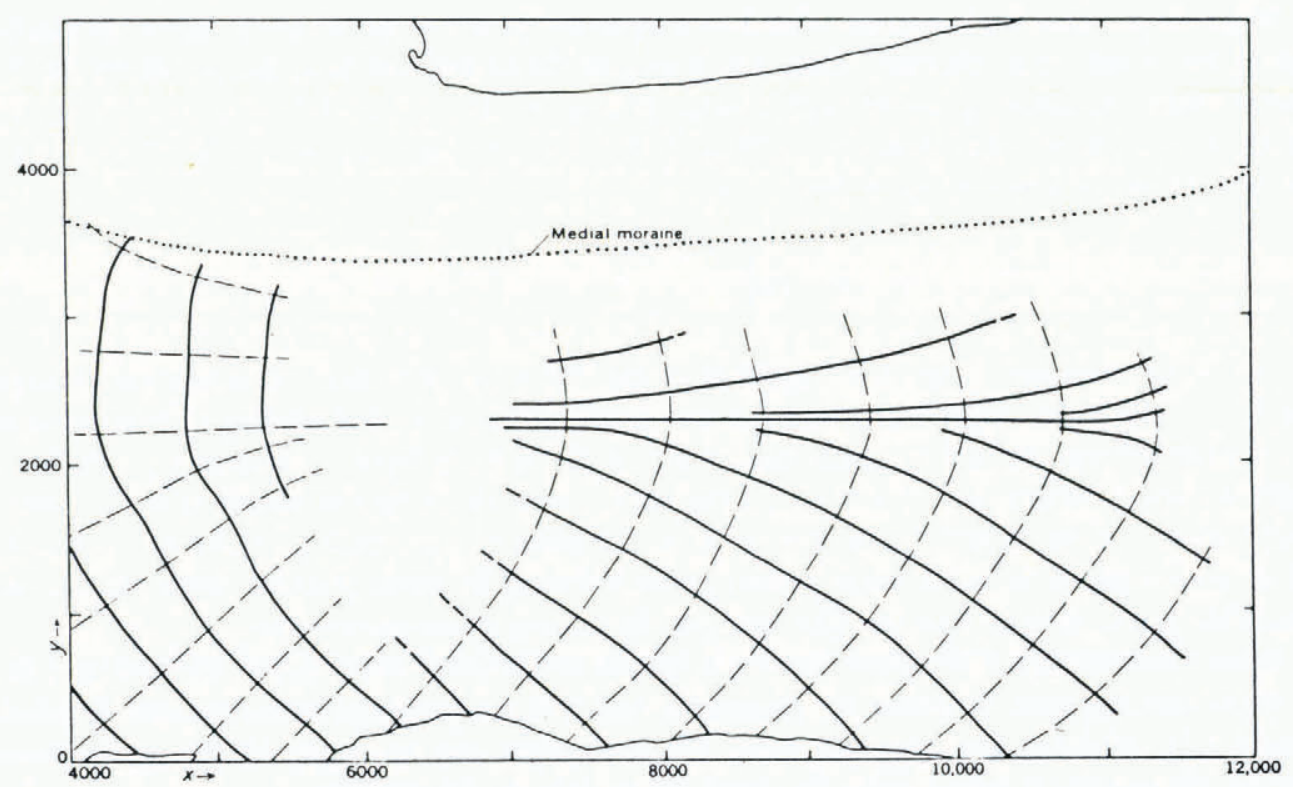

Fig. 3. Strain-rate trajectories measured on Saskatchewan Glacier, Alberta, Canada by Meier (1960, fig. 34). Full and broken lines correspond respectively to the algebraically lesser and greater principal strain-rate. (Reproduced by permission of Dr M. F. Meier.)

A generalized umbilic point is one where the velocity gradient tensor

$$
\boldsymbol{L}=\left(\begin{array}{ll}
u_{x} & u_{y} \\
v_{x} & v_{y}
\end{array}\right) \quad \text { takes the form } \quad\left(\begin{array}{rr}
p & q \\
-q & p
\end{array}\right) .
$$

Here $u, v$ are velocity components and subscripts denote derivatives. The conditions are then $u_{x}=v_{y}$ and $u_{y}=-v_{x}$, which are also precisely the conditions $\dot{\varepsilon}_{x x}=\dot{\varepsilon}_{y y}$ and $\dot{\varepsilon}_{x y}=0$ for an isotropic point for strain-rate. Thus, an isotropic point for strain-rate is also a generalized umbilic point.

To classify generalized umbilic points Thorndike and others (1978) consider the eigenvectors and eigenvalues of the symmetric tensor $\mathbf{L}^{\mathrm{T}} \mathbf{L}$, the directions of the eigenvectors being denoted by $\mathbf{a}, \mathbf{a}_{\perp}$, following Whitney (1955). The essential point is that the pattern formed by $\mathbf{a}, \mathbf{a}_{\perp}$ is different from the one formed by the principal directions of strain-rate $\dot{\varepsilon}=\frac{1}{2}\left(\boldsymbol{L}+\boldsymbol{L}^{\mathrm{T}}\right)$. Either pattern can be used with equal validity to classify the point (and the discriminant forms given by Thorndike and others (1978) apply for any symmetric tensor). In the sea-ice application it was convenient to use the $\mathbf{a}, \mathbf{a}_{\perp}$ pattern because of its close connection with cusps in the velocity map, while in the glacier application of this paper it seems more natural to use the pattern of principal strain-rate directions. The physical difference is, of course, that the tensor $\mathbf{L}^{\mathrm{T}} \boldsymbol{L}$ incorporates the rotation while the strain-rate tensor does not. Mathematically, $\boldsymbol{L}^{\mathrm{T}} \boldsymbol{L}$ and $\frac{1}{2}\left(\boldsymbol{L}+\boldsymbol{L}^{\mathrm{T}}\right)$ are simply two different ways of constructing a symmetric tensor from the unsymmetric $\boldsymbol{L}$.

The question remains: when we have found an isotropic point for strain-rate does its classification depend on whether we use $\boldsymbol{L}^{\mathrm{T}} \boldsymbol{L}$ or $\frac{1}{2}\left(\boldsymbol{L}+\boldsymbol{L}^{\mathrm{T}}\right)$ ? A detailed (and tedious) analysis 
shows that the index of the point does not in fact depend on which alternative we use, but both its line property and contour property do. Fundamentally this is because the line and contour properties can change continuously, while the index cannot. When $\boldsymbol{L}$ is symmetric all three properties are independent of which tensor we use; switching on an increasing antisymmetry in $\boldsymbol{L}$ cannot change the index but it can, and does, change the line and contour properties. Thus a particular point could, for example, be monstar for strain-rate but lemon for its pattern of $\mathbf{a}, \mathbf{a}_{\perp}$ directions.

For the stress this complication does not arise; the stress tensor is already symmetric and so there is no question of which symmetric tensor to use.

\section{Points OF PURE SHEAR}

A point in a two-dimensional velocity field where $\boldsymbol{L}$ has the form $\left(\begin{array}{cc}A & B \\ B & -A\end{array}\right)$ is defined by Thorndike and others (1978) as a generalized anti-umbilic point. This represents pure shear (with no rotation). On a glacier surface the two conditions on the tensor components thus implied will be satisfied at isolated points, and such points would be expected to occur on the centre-line. Of course, the points are not isotropic for strain-rate, the principal directions being well defined, but the tensor $\boldsymbol{L}^{\mathrm{T}} \boldsymbol{L}$ does have isotropic form, namely

$$
\boldsymbol{L}^{T} \boldsymbol{L}=\left(\begin{array}{cc}
A^{2}+B^{2} & 0 \\
0 & A^{2}+B^{2}
\end{array}\right)
$$

A map of the trajectories of its principal directions will thus show isotropic points not only at the isotropic points for strain-rate but also at the points of pure shear. By using $\boldsymbol{L}^{\mathrm{T}} \boldsymbol{L}$ the points of pure shear can be classified by line pattern and contour in exactly the same way as the isotropic points for strain-rate; there are accordingly six different possible kinds. Their positions and types provide further information about the essential structure of the field of strain-rate; as structurally stable features they could be used, just like the isotropic points, for making comparisons between observed and computed fields.

MS. received 4 March 1982 and in revised form 23 June 1982

\section{REFERENCES}

Berry, M. V., and Hannay, J. H. 1977. Umbilic points on Gaussian random surfaces. Journal of Physics, A: Mathematical and General, Vol. 10, No. 11, p. 1809-21.

Hambrey, M. J., and others. 1980. Dynamics and structure of Griesgletscher, Switzerland, by M. J. Hambrey, A. G. Milnes, and H. Siegenthaler. Journal of Glaciology, Vol. 25, No. 92, p. 215-28.

Meier, M. F. 1960. Mode of flow of Saskatchewan Glacier, Alberta, Canada. U.S. Geological Survey. Professional Paper 351 .

Nye, J. F. 1981. Structure and events in flow fields. (In Balian, R., and others, ed. Physique des défauts. Les Houches, session XXXV, 28 juillet-29 août 1980. Édite par R. Balian, M. Kleman, J.-P. Poirier. Amsterdam, etc., NorthHolland Publishing Co., p. 551-68.)

Thorndike, A. S., and others. 1978. The structure and evolution of flow fields and other vector fields, by A. S. Thorndike, C. R. Cooley, and J. F. Nye. Journal of Physics, A: Mathematical and General, Vol. 11, No.8, p. 1455-90

Whitney, H. 1955. Mappings of the plane into the plane. Annals of Mathematics, Vol. 62, No. 3, p. 374-410. 